\title{
Primary Gastric Adenosquamous Carcinoma: A Case Report
}

\author{
Ragia Aly ${ }^{\mathrm{a}}$, Sachin Gupta ${ }^{\mathrm{b}, \mathrm{g}}$, Sorab Guptac ${ }^{\mathrm{c}}$, Navroop Nagra ${ }^{\mathrm{d}}$, \\ Abhinav Goyale, Balraj Singh ${ }^{\mathrm{f}}$
}

\begin{abstract}
Gastric adenosquamous carcinoma (GASC) is a rare variant of gastric cancer; the limited data available suggest that these tumors are more aggressive and have a worse outcome in comparison to gastric adenocarcinoma. Currently, the management of GASC is similar to adenocarcinoma. We report a case of GASC that progressed rapidly despite treatment.
\end{abstract}

Keywords: Adenosquamous carcinoma; Stomach; Pembrolizumab; Adenocarcinoma; Gastric cancer; Stomach cancer

\section{Introduction}

It is estimated that about 27,000 new cases of gastric cancers will be diagnosed in the USA in the year 2020 [1]. Gastric adenocarcinoma (GAC) is the most common type of stomach cancer, constituting more than $90 \%$ of cases [2]. Gastric adenosquamous carcinoma (GASC) is a very rare variant of gastric cancer, accounting for less than $1 \%$ of cases [3]. GASC is more aggressive and carries a worse prognosis compared to GAC [4]. Given the rarity of GASC, limited data are available to draw conclusions regarding the optimal management of these patients. The current National Comprehensive Cancer Network (NCCN) guidelines for the management of stomach cancer mainly target GAC [5]. Patients with GASC are treated in accordance with the NCCN guidelines for GAC. We present a case of GASC that rapidly progressed despite treatment.

Manuscript submitted July 2, 2020, accepted July 29, 2020

Published online August 14, 2020

aDanbury Hospital, 24 Hospital Ave, Danbury, CT 06810, USA

bTower Health Reading Hospital, POBox16052, Reading, PA 19612-6052, USA

'Hematology Oncology, Einstein Healthcare Network, 5501 Old York, Philadelphia, PA 19141, USA

${ }^{\mathrm{d} H e p a t o l o g y, ~ V i r g i n a ~ M a s o n ~ M e d i c a l ~ C e n t e r, ~ S e a t t l e, ~ W A, ~ U S A ~}$

${ }^{\mathrm{e} G a s t r o e n t e r o l o g y, ~ E i n s t e i n ~ H e a l t h c a r e ~ N e t w o r k, ~} 5501$ Old York, Philadelphia, PA 19141, USA

fHematology Oncology, St. Joseph's University Medical Center, 703 Main St, Paterson, NJ 07503, USA

gCorresponding Author: Sachin Gupta, Tower Health Reading Hospital, P.O. Box 16052, Reading, PA 19612-6052, USA. Email: Docgupta1@gmail.com

doi: https://doi.org/10.14740/gr1307

\section{Case Report}

A 61-year-old man with a medical history of hypertension, pre-diabetes, osteoarthritis, and seizures presented with hematemesis and melena. He also reported poor appetite and unintentional $6.5 \mathrm{~kg}$ weight loss. On physical exam, he had notable pallor; the rest of his exam was within normal limits. His blood work was unremarkable except for low hemoglobin of $8.2 \mathrm{~g} / \mathrm{dL}$ (reference range: $14-16 \mathrm{~g} / \mathrm{dL}$ ). He underwent esophagogastroduodenoscopy (EGD), which revealed a large (approximately $5 \mathrm{~cm}$ ) fungating, ulcerating, partially necrotic antral mass. Biopsies obtained during the EGD showed poorly differentiated carcinoma with immunohistochemical stains consistent with squamous cell carcinoma. The patient underwent subtotal gastrectomy with Billroth II antecolic gastrojejunostomy. Histopathological examination of the tumor was consistent with squamous cell carcinoma in solid areas of the mass. Additionally, both well and poorly differentiated adenocarcinoma components were also identified with glandular and papillary formation. The tumor was characterized as GASC. The omental margin was involved, and three of five lymph nodes were positive for the squamous cell component. On immunohistochemistry testing, there was no loss of expression of DNA mismatch repair proteins MLH1, PMS2, MSH2, and MSH6. It was also HER-2 negative. According to the American Joint Committee on Cancer (AJCC) staging, the patient stage was pT4a N2 M0. Adjuvant chemoradiation was recommended. He was started on 5-fluorouracil (5FU) and 3-dimensional (3D)-external beam radiation. Before completing the combined chemoradiation therapy, the patient presented to the emergency department for persistent hiccups. Imaging studies revealed a mesenteric mass and liver metastasis. The patient underwent a liver biopsy, which showed poorly differentiated squamous cell carcinoma (immunohistochemical stains positive for $\mathrm{p} 63$, p40, and CK5/6). The patient was started on folinic acid, fluorouracil, and oxaliplatin (FOLFOX) regimen. Follow-up computed tomography (CT) scan after four cycles of FOLFOX chemotherapy showed an increase in the size and number of the metastatic lesions. The patient was switched to palliative chemotherapy with paclitaxel and ramucirumab. Ramucirumab was discontinued after a single dose as the patient had a transient ischemic attack (TIA). Paclitaxel was continued; however, the patient's performance status deteriorated and was deemed not a chemotherapy candidate. Testing for programmed death ligand-1 (PD-L1) showed a combined positive score (CPS) of 10. The plan was to start pembrolizumab. Unfortunately, the patient 
passed away before the start of treatment.

\section{Discussion}

The diagnosis of GASC is based on the presence of both adenocarcinoma and squamous cell carcinoma on histopathological examination, with the squamous cell component constituting at least $25 \%$ of the tissue [3]. The origin of the squamous cell carcinoma component is not fully explained. Multiple possibilities have been suggested, including the metaplastic transformation of adenocarcinoma, origination from mucosal stem cells, or the fusion of coexisting squamous cell carcinoma and adenocarcinoma [6]. Most cases of GASC are locally advanced and have lymph node involvement or distant metastasis at the time of diagnosis. Ge et al, in a retrospective cohort study, concluded that patients with GASC had higher rates of poorly differentiated tumors and a higher rate of metastasis, and more than $15 \%$ of patients had a tumor that was at T4 stage [7]. In another retrospective study done by Akce et al, the patients with GASC and squamous cell carcinoma had statistically significant lower overall survival when compared to patients with adenocarcinoma [8]. This was similar to what was concluded by Ge et al in their study [7]. It has been suggested that the behavior of the tumor depends mainly on the adenocarcinoma component rather than the squamous cell component [9]. However, this has not been confirmed due to the small number of cases. In the study done by Fang et al looking at the data of 120 patients with adenosquamous carcinoma, patients with metastatic disease had lesions originating from both the glandular and the squamous components [4]. In the three mentioned studies, there was no difference in the management regimen when compared to patients with adenocarcinoma but there were worse outcomes $[4,7,8]$. In the last few years, the field of immunotherapy has shown a real promise in revolutionizing the management of multiple solid tumors at advanced stages with fewer adverse effects. This was demonstrated in multiple clinical trials, most famously the KEYNOTE trials studying the programmed death-1 (PD-1) inhibitor, pembrolizumab as monotherapy, or in combination with chemotherapy for treatment of multiple cancers [10-12]. Pembrolizumab has been studied in multiple clinical trials for the treatment of advanced gastric and gastroesophageal adenocarcinoma in patients who failed previous lines of treatments $[11,13,14]$. The current NCCN guidelines include pembrolizumab as a second line for microsatellite instability (MSI) or as a third-line or subsequent therapy option for patients with locally advanced or metastatic GAC who have PD-L1 expression with CPS score greater than or equal to $1[5]$.

\section{Conclusions}

The poor outcome associated with GASC could be attributed to the innate aggressive nature of these tumors with a higher likelihood of serosal invasion, early lymph node involvement or the presence of distant metastasis at the time of diagnosis. Moreover, squamous component may render these tumors less responsive to the currently available chemotherapy regimens. However, the small number of cases in the available retrospective studies renders the results inconclusive. Further studies are needed to characterize this aggressive cancer and optimize the treatment plans.

\section{Acknowledgments}

None to declare.

\section{Financial Disclosure}

None to declare.

\section{Conflict of Interest}

None to declare.

\section{Informed Consent}

Written Consent was obtained from patient's family after his death.

\section{Author Contributions}

RA provided the idea of the Case Report and contributed to the writing of it; Sachin Gupta and Sorab Gupta contributed to the writing of the Case Report; NN and AG contributed to the editing of the Case Report; BS was responsible for the supervision.

\section{Data Availability}

The authors declare that data supporting the findings of this study are available within the article.

\section{References}

1. American cancer society, Cancer facts and figures 2020 .

2. Karimi P, Islami F, Anandasabapathy S, Freedman ND, Kamangar F. Gastric cancer: descriptive epidemiology, risk factors, screening, and prevention. Cancer Epidemiol Biomarkers Prev. 2014;23(5):700-713.

3. Chen H, Shen C, Yin R, Yin Y, Chen J, Han L, Zhang B, et al. Clinicopathological characteristics, diagnosis, treatment, and outcomes of primary gastric adenosquamous carcinoma. World J Surg Oncol. 2015;13:136.

4. Feng F, Zheng G, Qi J, Xu G, Wang F, Wang Q, Guo M, et al. Clinicopathological features and prognosis of gastric adenosquamous carcinoma. Sci Rep. 2017;7(1):4597.

5. NCCN Guidelines Version 2.2020 Gastric Cancer. https:// www.nccn.org/professionals/physician_gls/pdf/gastric. 
pdf.

6. Yoshida K, Manabe T, Tsunoda T, Kimoto M, Tadaoka Y, Shimizu M. Early gastric cancer of adenosquamous carcinoma type: report of a case and review of literature. Jpn J Clin Oncol. 1996;26(4):252-257.

7. Ge Y, Lin L, Ma X, Luo D, Shi L, Jiang M, Fan H, et al. Adenosquamous Carcinoma of the Stomach: A Population-based Study from the SEER Database. J Cancer. 2019;10(23):5705-5713.

8. Akce M, Jiang R, Alese OB, Shaib WL, Wu C, Behera $\mathrm{M}$, El-Rayes BF. Gastric squamous cell carcinoma and gastric adenosquamous carcinoma, clinical features and outcomes of rare clinical entities: a National Cancer Database (NCDB) analysis. J Gastrointest Oncol. 2019;10(1):85-94.

9. Mori M, Iwashita A, Enjoji M. Adenosquamous carcinoma of the stomach. A clinicopathologic analysis of 28 cases. Cancer. 1986;57(2):333-339.

10. Kato K, Shah MA, Enzinger P, Bennouna J, Shen L, Adenis A, Sun JM, et al. KEYNOTE-590: Phase III study of first-line chemotherapy with or without pembrolizumab for advanced esophageal cancer. Future Oncol. 2019;15(10):1057-1066.
11. Fuchs CS, Doi T, Jang RW, Muro K, Satoh T, Machado M, Sun W, et al. Safety and efficacy of pembrolizumab monotherapy in patients with previously treated advanced gastric and gastroesophageal junction cancer: phase 2 clinical KEYNOTE-059 trial. JAMA Oncol. 2018;4(5):e180013.

12. Langer CJ, Gadgeel SM, Borghaei H, Papadimitrakopoulou VA, Patnaik A, Powell SF, Gentzler RD, et al. Carboplatin and pemetrexed with or without pembrolizumab for advanced, non-squamous non-small-cell lung cancer: a randomised, phase 2 cohort of the open-label KEYNOTE-021 study. Lancet Oncol. 2016;17(11):1497-1508.

13. Shitara K, Ozguroglu M, Bang YJ, Di Bartolomeo M, Mandala M, Ryu MH, Fornaro L, et al. Pembrolizumab versus paclitaxel for previously treated, advanced gastric or gastro-oesophageal junction cancer (KEYNOTE-061): a randomised, open-label, controlled, phase 3 trial. Lancet. 2018;392(10142):123-133.

14. Muro K, Chung HC, Shankaran V, Geva R, Catenacci D, Gupta S, Eder JP, et al. Pembrolizumab for patients with PD-L1-positive advanced gastric cancer (KEYNOTE-012): a multicentre, open-label, phase $1 \mathrm{~b}$ trial. Lancet Oncol. 2016;17(6):717-726. 